\title{
Study on predistortion techniques and adaptive modulation used in fading channels
}

\author{
Wei Liu \\ School of Physics and Electronic Engineering \\ Harbin Normal University \\ Harbin, China \\ e-mail: ljliuwei@yeah.net
}

\author{
E Zhang \\ School of Physics and Electronic Engineering \\ Harbin Normal University \\ Harbin, China \\ e-mail: hsdzhe@126.com
}

\begin{abstract}
Spectral efficiency is one of primary concern in the design of any wireless data communications systems, where fading is the main problem. An approach that can be taken to overcome the problem of fading is to adapt the modulation scheme according to the instantaneous propagation channel conditions. In this paper, we present a simplified mathematical model to calculate the probability of SER for any given constellation of M-QAM. This model plays an important role in designing spectrally efficient adaptive modulation over nonlinear mobile channels. Instantaneous spectral efficiency and its histogram distribution are found for linear and nonlinear channel cases. The achievable spectral efficiency is obtained for linear and nonlinear channel and compared with non-adaptive system and Shannon capacity.
\end{abstract}

Keywords- predistortion techniques; adaptive modulation; fading channels

\section{INTRODUCTION}

All first generation cellular systems used analog frequency modulation. However, a pressing need for greater bandwidth efficiency has led to the use of digital modulation techniques in second-generation cellular systems. Now as the demand for multimedia services grows, the ability to provide spectrally efficient and flexible data rate access are an important design considerations for satellite systems, as well as for the third and forth generation wireless systems where high power efficiency is needed. Spectral and power efficiencies are the main two challenges for future wireless communication systems. Spectral efficiency represents the ability of a system (e.g. modulation scheme [1-2]) to accommodate data within an allocated bandwidth. In addition to that, wireless systems suffer from fading due to the time-varying nature of the propagation channel [3]. Adaptive modulation is an attractive solution to improve the spectral efficiency in fading environment by adapting the modulation scheme and the transmitted power according to instantaneous propagation conditions, interference scenario, and traffic or data rate requirements. Equalization and predistortion are the viable techniques to overcome the nonlinearity problems [4]. Equalization is usually done at the receiver side. The main function of the equalizer is to equalize the distorted received signal and thus lowers the Symbol Error Rate (SER) to an acceptable level. Predistortion technique on other hand is usually done at the transmitter side.
Although demand for wireless services is growing at a rapid pace, the availability of the radio spectrum for such services remains extremely scarce. Adaptive modulation techniques have been recently proposed for two-way data transmission over cable. Similar ideas have also been applied to improve the BER on mobile channels. For instance, variable rate QAM has been proposed for several third generation wireless communication systems. When the channel characteristics are estimated and sent back to the transmitter, the transmission scheme can be adapted relative to the estimate [5]. Most of traditional modulation and coding techniques do not adapt to fading or nonlinear conditions. These non-adaptive methods require a fix-link margin to maintain acceptable performance when the channel quality is poor. Thus, these systems are effectively designed for the worst-case channel conditions, resulting in insufficient utilization of the full channel capacity. Adapting to signal fading allows the channel to be used more efficiently since the power rate can be adjusted to take advantage of favorable channel conditions [6]. In particular the Shannon capacities of three adaptation policies were compared: optimal simultaneous power and rate adaptation, constant power with optimal rate adaptation, and channel inversion with a fixed rate. Results indicated that rate adaptation is the key parameter controlling the link spectral efficiency. Rate adaptation can be achieved through variation in symbol time duration or constellation size.

The objective of this paper is to provide a tractable mathematical model for evaluating the Symbol Error Rate (SER) of circular and rectangular M-QAM constellations over nonlinear channel. Then by using a Gray coding, which is an easy and efficient coding technique for two dimensional constellations, the BER can approximately be found by dividing the SER by the number of bit per symbol.

\section{PRobability OF ERROR CALCULATION IN NONLINEAR COMMUNICATION SYSTEMS}

Based on Bit Error Rate (BER) performance of the different M-QAM constellations over an AWGN channel, adaptive modulation provides high average spectral efficiency by transmitting at a high speed under favorable channel conditions, and reducing throughput as the channel degrades [7]. The probability of error, given any other symbol in the constellation is transmitted, can be obtained similarly. The exact average probability of symbol error for M-ary data in AWGN is the weighted sum of probabilities 
for all sub-regions of every possible signal point which can be calculated as:

$$
\begin{aligned}
& P_{e}=\sum_{i=1}^{M_{i}} p\left(e / x_{i}\right) P\left(x_{i}\right)=\sum_{i=1}^{M} \sum_{j=1}^{G} \frac{P\left(x_{i}\right)}{2 \pi} \cdot \\
& \int_{0}^{\eta_{j, i}} \exp \left(-\frac{l_{j, i}^{2} \sin ^{2} \psi_{j, i}}{N_{0} \sin ^{2}\left(\theta+\psi_{j, i}\right)}\right) d \theta
\end{aligned}
$$

where $P\left(x_{i}\right)$ is the prior probability of transmitted symbol $x_{i}, \psi_{j, i}$ and $\eta_{j, i}$ are parameters corresponding to sub-region $j$, as defined in Figure 1 , and $G$ is the total number of erroneous sub-regions for $X_{i}$ in the constellation. In equation (1), $l_{j, i}^{2} / N_{0}$ is a function of $\gamma$, where $\gamma$ is the SNR, and it can be expressed as: $l_{j, i}^{2} / N_{0}=b_{j} \gamma$, where $b_{j}$ is a constant for the constellation. Then, the probability of error can be written as a function of $\gamma$, i.e:

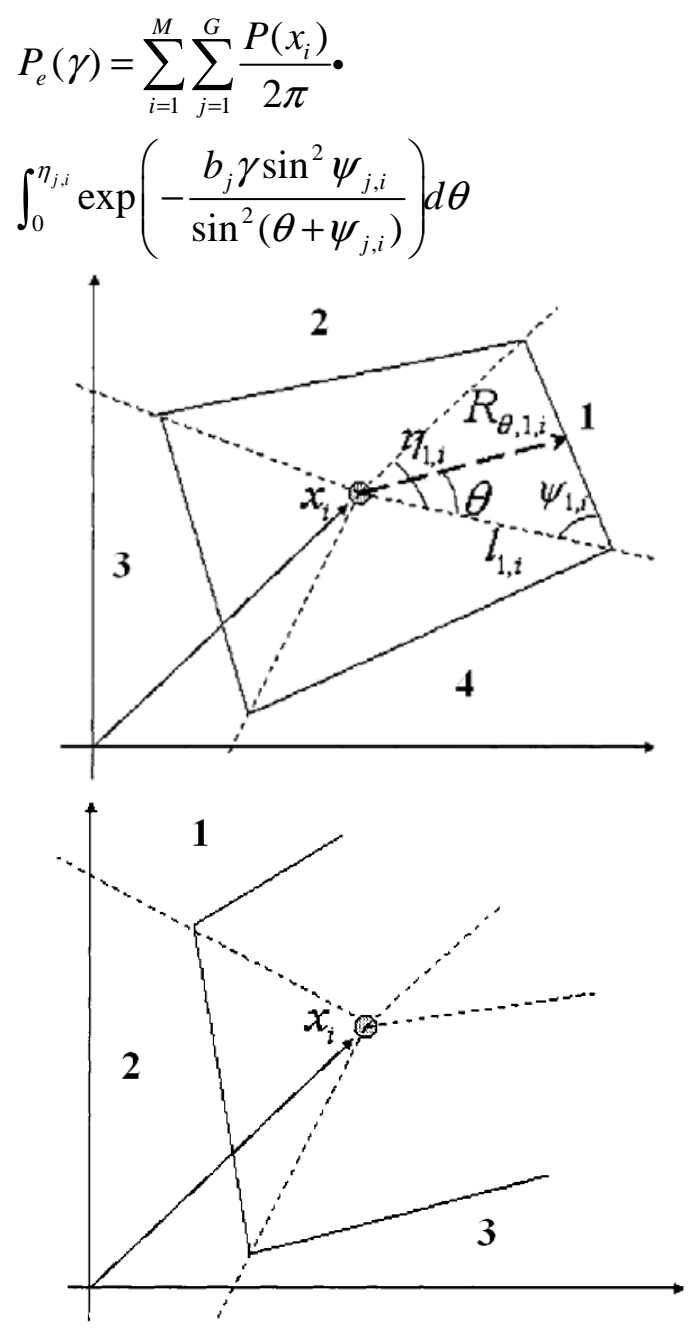

Figure 1. Decision boundaries of 2-D signal constellation
From the previous section, we see that method calls for a clear knowledge of the decision boundaries of all constellations. For nonlinear systems this becomes a formidable task. Moreover, Craig's method needs numerical integration to find out the average SER. To overcome these shortfalls, in this section and the following one, we propose a simplified approach to evaluate the bounded SER, which does not need either the numerical integration or the knowledge of the decision regions. We discuss this simplified methodology for finding out the SER of different 16-QAM circular constellations. Here, after presenting the undistorted and the distorted structures of each constellation after passing the HPA, we discuss the analytical technique used to derive the bounded SER expression. For better explanation the geometry parameters and its distances, each constellation will be presented for undistorted constellation. The same parameters and the analysis are applicable for the distorted case. The analysis, which is dependent on the geometry of the different constellations, provides optimal RRs and PDs for different constellations. These optimal values are found in the sense that with these geometrical values, each constellation will give a minimum SER performance.

\section{PROBABILITY OF ERROR ANALYSIS}

The analytical technique to find the SER, presented in this section, is dependent on the geometry of the constellation diagram. Here, as a result of symmetry of the symbol orientations on both circles, we can arbitrarily choose any two symbols, one from each circle, for the analysis. Now, if we transmit any arbitrary symbol from the transmitter in the presence of AWGN of one-sided PSD $N_{0}$, the lower bound of the SER could be given as,

$$
S E R_{\text {lower }}=Q \sqrt{\frac{d_{\text {min }}^{2}}{2 N_{0}}}
$$

where $d_{\text {min }}$ represents the minimum distance among all the distances. From the expressions of the distance parameters it is clear that $d_{\min }$ is either a function of RR, $\mathrm{PD}$ and symbol energy or a function of symbol energy only. It is worthwhile to mention that the symbol energy itself is a function of the RR. We can also express the upper bound for the SER in terms of $d_{\text {min }}$, which will be given as,

$$
S E R_{\text {weakupper }}=15 Q \sqrt{\frac{d_{\min }^{2}}{2 N_{0}}}
$$

where the factor 15 represents the number of neighboring symbols around the transmitted one. For calculating the bounded SER for the transmitted symbol in the AWGN, we can carry out the following procedure. First, assuming that one presence of the outer circle symbols is transmitted, we calculate the corresponding SER and then assuming that one of the inner circle symbols is transmitted, we calculate the corresponding SER. Finally we average the above SER expressions over the PDF of the symbols. Assuming all the 
symbols are equally-likely, we get that the probability of any symbol either in the outer or inner circle. As a result, the bounded SER expression becomes:

$$
\begin{aligned}
& \operatorname{SER}_{(8,8)}=\sum_{i=1}^{8} Q \sqrt{\frac{d_{A_{1} B_{i}}^{2}}{2 N_{0}}} \\
& +\frac{1}{2} \sum_{i=2}^{8} Q \sqrt{\frac{d_{A_{1} A_{i}}^{2}}{2 N_{0}}}+\frac{1}{2} \sum_{i=2}^{8} Q \sqrt{\frac{d_{B_{1} B_{i}}^{2}}{2 N_{0}}}
\end{aligned}
$$

Equations (3-5) provide generalized SER expressions for the circular $(8,8)$ constellation, which are equally applicable for both linear and nonlinear environments. The expressions can easily be evaluated by calculating the constellations' distances.

\section{RESUlts AND DisCUSSIONS}

In this section we will present some analytical results based on the findings in the previous subsections, taking into account the effects of constellation's RR and PD on the SER performance. Some supporting simulation results are also presented here. Moreover, this section provides a brief performance comparison between different 16-QAM circular constellations presented in the above subsections. The presented results are based on the base-band nonlinear communication system model shown in Figure.2.

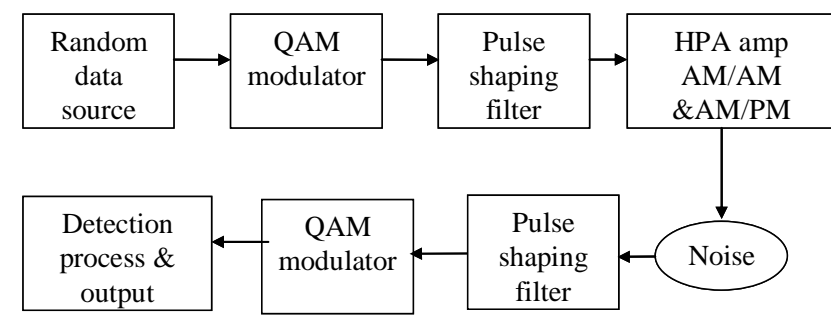

Figure 2. Nonlinear communication system

We used the base-band model because it requires less computation in the simulation cycle. The function of each component of the system model has been described in chapter two. Here we assume that the system has no ISI and $\mathrm{ACI}$ and the transmitter and receiver filters are Nyquist pulse shaping filters.

Figure.3 shows the effect of various RRs on the SER performance of the circular $(8,8)$ QAM constellation in the presence of the HPA and a predicted optimum PD of $22.5^{\circ}$. This $22.5^{\circ} \mathrm{PD}$ maximizes the distances between the inner circle symbols and the outer circle symbols (the range of PD is between $0^{\circ}$ to $45^{\circ}$ ). With this value, any inner circle symbol positions itself at an equidistant length from its two neighboring outer circle symbols providing the best possible structure for detecting any one of the symbols. In the figure, the effects of the RR are observed for different SNRs in $\mathrm{dB}$. From the figure it is found that the SER performance continues to improve with continuous increase in the RR up to its optimal value, while an increase in the RR beyond its optimal value results in worse SER performance.

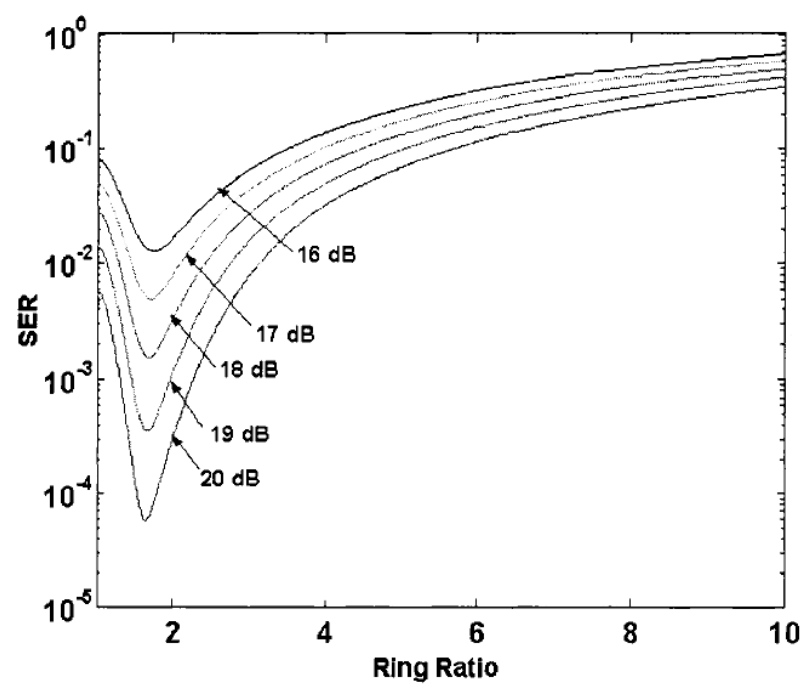

Figure 3. Effect of RR on the SER performance of circular $(8,8)$ constellations

There is an explanation behind this particular observation. In the beginning $(R R=1)$ all the constellation points lie on the same circle providing a very small decision region for detection. With the assumption that the maximum amplitude of the symbols is normalized to 1 as the RR is increased, the decision region becomes wider for detection with a decrease in the radius of the inner circle. As a result the performance keeps improving until the RR reaches its optimum value (having lowest SER). After the optimal RR is reached, the constellation points in the inner circle become so crowded that the decision region in this circle becomes very small for detection purposes. As a result the system performance starts to degrade. From this Figure we can see that the optimal RR is change slightly when the SNR is change.

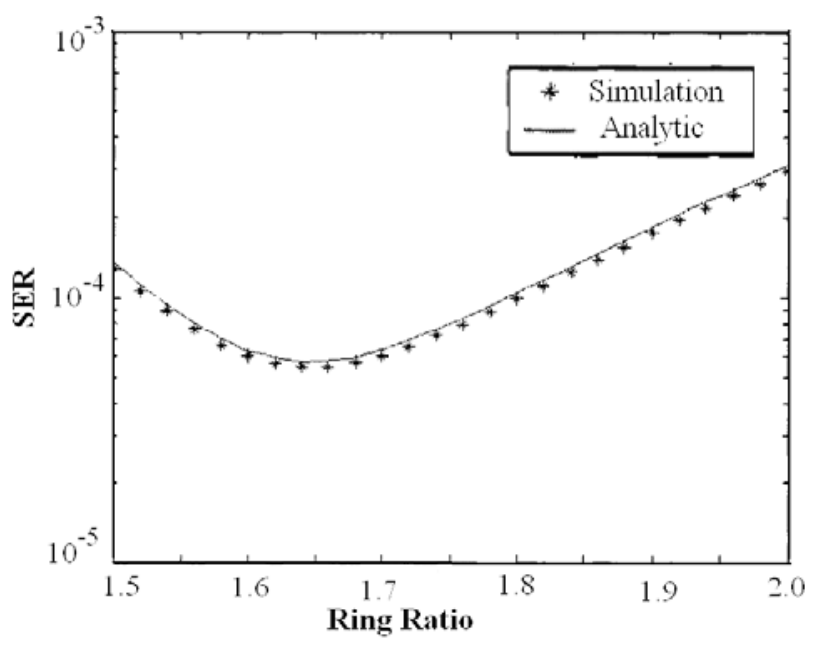

Figure 4. Analytical and simulation results: SER versus PR of $(8,8)$ constellations 
The plots also show that the SER performance is asymmetrical with respect to the optimal RR. This results in a large degradation for a small deviation in the RR from its optimal value. To check the accuracy of the analytical results, Figure.4 compares the analytical results with respect to computer simulations for the $(8,8)$ constellation with average SNR of $20 \mathrm{~dB}$. It is clear that the simulation results are closely agree with the analytical results.

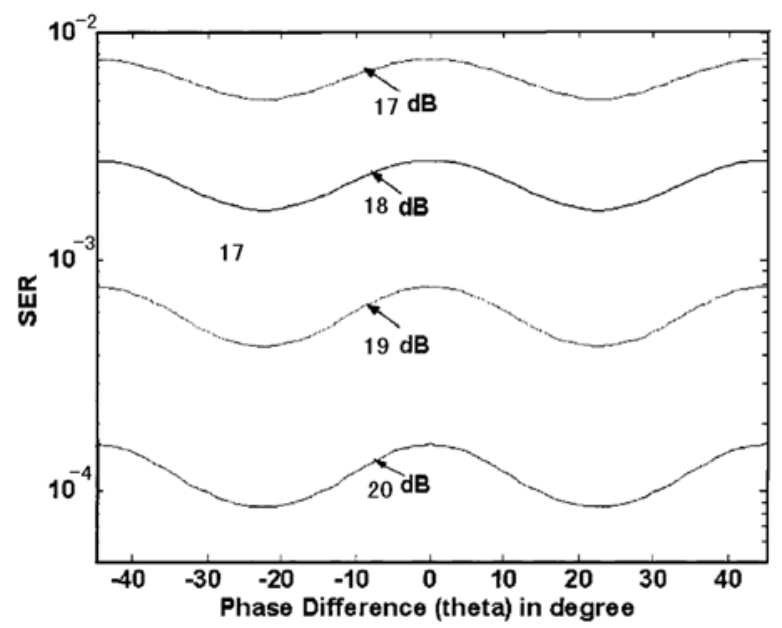

Figure 5. Effect of PD on the SER performance of circular $(8,8)$ constellations

Figure.5 presents the effect of various PDs on the SER performance for the circular $(8,8)$ QAM constellation. Here, the effects of PDs are observed with different SNRs in $\mathrm{dB}$ while the optimal RR is fixed to 1.645. In this figure the optimal PD is shown to be $22.5^{\circ}$ which is obvious since the optimal RR, based on which the PDs are plotted, was achieved by predicting the above value.

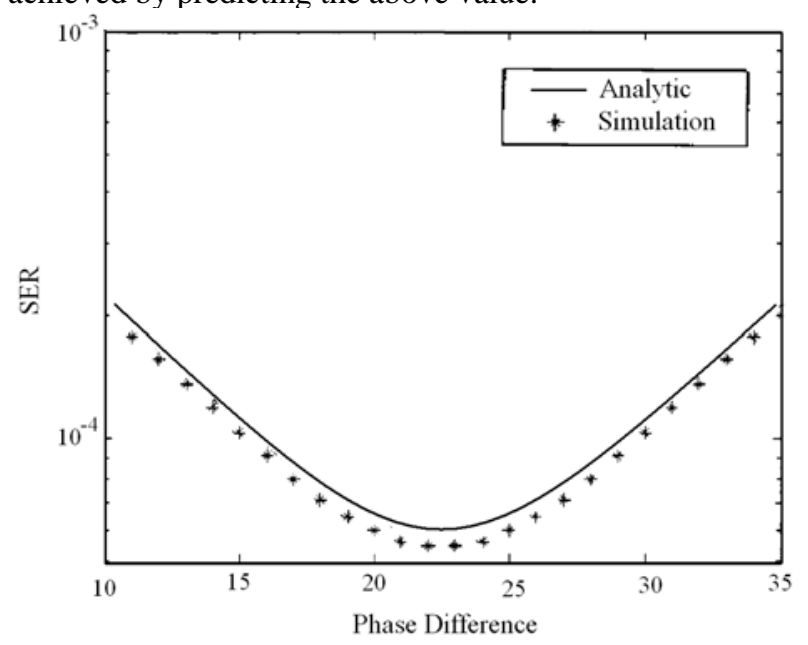

Figure 6. Analytical and simulation results: SER versus PD of $(8,8)$ constellations

In this case, it is found that if we vary the optimal RR to some extent, the optimal PD does not vary, which proves the validity of its value. In the figure, this optimal PD is shown to be independent of the SNRs. The figure also shows that the SER performance is symmetrical with respect to the optimal PD. Figure. 6 shows the analytical results vs. simulation results, of SER performance versus PD for $(8,8)$ constellation. Again the simulation results are closely agreed with the analytical results.

\section{CONCLUSION}

This paper has presented a simple and efficient method to calculate the bounded SER based on the constellation's geometry. This method can be applied for linear and nonlinear channel cases. Moreover this method, which can be extended for any M-QAM circular constellation, provides a way to find the optimal RRs and the optimal PDs for all the addressed QAM constellations. The results have shown that the $\mathrm{RR}$ has more influence than the $\mathrm{PD}$ on the variation of the SER performance. In addition to that, computer simulations have been used to support the theoretical results. Finally, the analytical results have been used to find the optimum RRs and PDs for all constellations. Results show that the RRs are having more effect on the SER than the PDs.

\section{REFERENCES}

[1] D. Divsalar, and E. Biglieri, "Upper bounds to error probabilities of coded systems beyond the cutoff rate" IEEE commun., vol. 51, pp.12011-2018, Dec. 2003.

[2] D. Divsalar, and E. Biglieri, "Upper bounds to error probabilities of coded systems over AWGN and fading channels" Proc.Global Telecommunications Conf.,2000. (GLOBECOM'00), vol. 3, pp. 1605-1610, 27 Nov-1 Dec. 2000.

[3] X. Dong, N.C. Beaulieu, and P.H. Wittke, "Signaling constellations for fading channels", IEEE Trans. Commun., vol. 47, pp. 703-714, May 1999

[4] P. Dong-Chul and J. J. Tae-Kyun, "Complex-bilinear recurrent neural network for equalization of a digital satellite channel", IEEE Trans. Neural Networks, vol. 13, pp. 711-725, May 2002.

[5] D. D. Falconer, "Adaptive equalization of channel nonlinearities in QAM data transmission systems" Bell Syst. Tech. J., vol. 57, pp. 2589-2611, Sept. 1978

[6] M. Filip and E. Vilar "Optimum utilization of the channel capacity of a satellite link in the presence of amplitude scintillations and rain attenuation", IEEE Trans. Commun., vol. 38, pp. 1958-1965, Nov. 1990.

[7] G. Forney Jr., R. Gallager, G. Lang, F. Longstaff, and S. Qureshi "Efficient modulation for band-limited channels", IEEE J. Select. Areas Commun., vol. SAC-2, pp. 632-647, Sept. 1984. 